\title{
Optimal Energy Aware Routing Path (OEARP) for Wireless Sensor Networks
}

\author{
http://dx.doi.org/10.3991/ijim.v9i1.4203 \\ A.S. Alkalbani ${ }^{1}$, A.O.M. Tap ${ }^{1}$ and T. Mantoro ${ }^{2}$ \\ ${ }^{1}$ International Islamic University Malaysia, Kuala Lumpur, Malaysia \\ ${ }^{2}$ University Siswa Bangsa International, Jakarta, Indonesia
}

\begin{abstract}
OEARP is a set of widely held routing protocols for Wireless Sensor Networks (WSNs). There are many issues and challenges that affect routing of data such as high energy consumption, which reduces network lifetime, and vulnerable routing path is major threat for sensor nodes. The aim of this paper is to propose an energy aware routing approach that enhances the energy levels of each sensor during cluster heads and routing path selections. The optimal energy aware routing path (OEARP) improves performance, network efficiency and maximize network lifetime. These were measured through simulations and the results were analyzed and compared with other well-known mechanisms including GLBCA, LBC, and EELBC.
\end{abstract}

Index Terms - WSNs, Network efficiency, Power Consumption, Routing Protocol, Energy Aware Routing Path.

\section{INTRODUCTION}

Due to recent developments in networks such as high performance, increased network security, minimum-cost, and low power consumption, multifunctional sensors have led to increased attention in this area. In the last few years, WSNs and related applications have gained significant momentum. In fact it is the main technology evolving away from research-driven environment into commercial interests [1].

WSNs use distributed sensors to monitor various conditions of remote locations such as smoke, sound, vibration, pressure, temperature, motion, and pollution. WSNs are configured autonomously by sensor nodes equipped with sensing, computing and wireless communication capabilities [2]. The main purpose of sensor nodes in wireless techniques is to collect the useful data and transmit those data back to the base station for possible use. Every node is capable of sensing, data processing, communication, and operates on its limited amount of battery energy consumed mostly in transmission and receives signals (receiver) at its radio transceiver [3]. Hence, the energy consumption of sensor nodes needs to be seriously considered to achieve longer surveillance [4]. A fundamental part in the WSNs configuration is how the sensors reciprocate data among the network until they reach to base station for data processing; a specialized routing protocol is required to manage transmission band. The design of such a routing protocol must take into consideration the limited resource utilization and security objectives. Numerous protocols have been designed in this area but few of them were constructed with care of the limited resource utilization, clustering and routing issues at the same time [5]. The main problem for this research is high energy consumption when transmitting data from source to base station and sensors rapidly dying, which render isolated parts of WSN useless.

This research paper proposes an optimized protocol, which considers energy limitation and routing issues for WSNs. This protocol mainly depends on nodes energy level for selecting Cluster Head $(\mathrm{CH})$ during network construction phase and energy aware routing path for transmitting packets from source node to Base Station (BS). This provides optimal connections between the sensor nodes and the sink node with minimal cost in terms of delay and power consumption.

In this research, the nodes are assumed as static. Hence the contributions are suitable for networks which are either static or whose topology changes slowly such that there is enough time for optimally balancing the traffic between successive topology changes.

The remaining part of the paper is organized as follows: In section 2, the related work in this area is given. Section 3 , describes the research framework model. Section 4, shows the mathematical representation of the research model. In section 5, network clustering construction and optimal energy aware routing algorithm steps are described. In section 6, extensive experiments of simulation are conducted to prove the efficiency and quality of the proposed model. Section 7, presents the research contribution, results discussion, and analysis. Conclusion as well as the challenges encountered and future directions for research is given in section 8 .

\section{RELATED WORK}

In the last decade, deep research showed the correlation between the sensors in data collection, data processing, managing the sensing activity and data transmission. Due to the limitations of sensor nodes in power and communication bandwidth, innovative mechanisms were proposed to minimize energy consumption that decreases WSNs lifetime and efficient use of the limited bandwidth. Many challenges are faced in construction and management of power efficient and secure routing protocols for WSNs due to the limitations and constraints such as the large number of sensors that are deployed, self-organizing requirement and energy limits [6].

WSNs routing protocols are categorized into two major types such as network structure and protocol operation. Each category is classified to many routing types as shown in figure 1 [7]. In this study, the hierarchical routing type is selected to study the influence on the 
lifetime of the network because the structure of this type is optimized and resources are efficiently utilized.

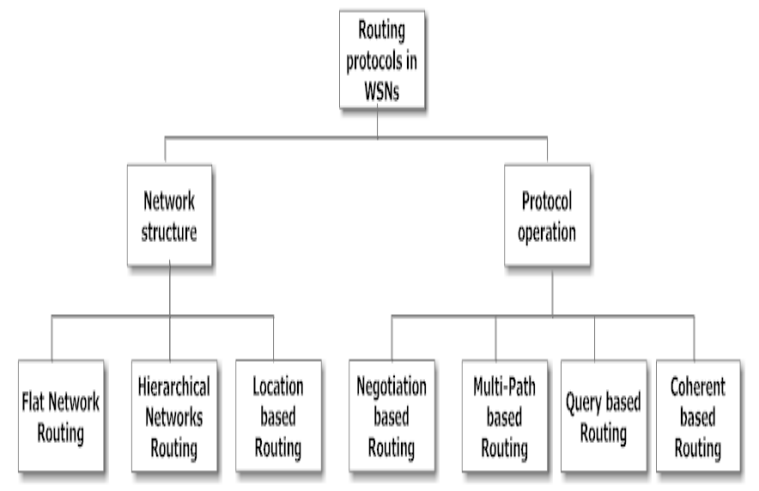

Figure 1. Categories of WSNs Routing Protocols

Reference [6] presented WSNs routing techniques classifications which describe advantages and performance issues of each technique as shown in Table I.

TABLE I.

CLASSIFICATION AND COMPARISON OF ROUTING PROTOCOLS IN WSNS

\begin{tabular}{|c|c|c|c|c|}
\hline $\begin{array}{c}\text { Routing } \\
\text { Protocols } \\
\end{array}$ & Classification & $\begin{array}{l}\text { Power } \\
\text { Usage }\end{array}$ & Scalability & $\begin{array}{l}\text { Over } \\
\text { head }\end{array}$ \\
\hline SPIN & $\begin{array}{l}\text { Flat / Source } \\
\text { initiated } \\
\text { /Data-centric }\end{array}$ & Ltd & Ltd & Low \\
\hline$\overline{\mathrm{DD}}$ & $\begin{array}{c}\text { Flat/Data } \\
\text { centric/Des- } \\
\text { initiated }\end{array}$ & Ltd & Ltd & Low \\
\hline $\mathrm{RR}$ & Flat & Low & Good & Low \\
\hline GBR & Flat & Low & Ltd & Low \\
\hline CADR & Flat & Ltd & Ltd & Low \\
\hline COUGAR & Flat & Ltd & Ltd & High \\
\hline ACQUIRE & $\begin{array}{c}\text { Flat/ } \\
\text { Data-centric }\end{array}$ & Low & Ltd & Low \\
\hline LEACH & $\begin{array}{c}\text { Hierarchical/ } \\
\text { Des- } \\
\text { initiated }\end{array}$ & High & Good & High \\
\hline $\begin{array}{l}\text { TEEN \& } \\
\text { APTEEN }\end{array}$ & Hierarchical & High & Good & High \\
\hline PEGASIS & Hierarchical & Max & Good & Low \\
\hline VGA & Hierarchical & Low & Good & High \\
\hline SOP & Hierarchical & Low & Good & High \\
\hline GAF & $\begin{array}{l}\text { Hierarchical/ } \\
\text { Location }\end{array}$ & Ltd & Good & Mod \\
\hline SPAIN & $\begin{array}{l}\text { Hierarchical/ } \\
\text { Data-centric }\end{array}$ & Ltd & Ltd & High \\
\hline GEAR & Location & Ltd & Ltd & Mod \\
\hline SAR & Data-centric & High & Ltd & High \\
\hline SPEED & $\begin{array}{c}\text { Location/ } \\
\text { Data-centric }\end{array}$ & Low & Ltd & Less \\
\hline
\end{tabular}

The energy conservation for WSNs was studied by proposing Conditional Max-Min Battery Capacity Routing (CMMBCR) [8]. The mechanism of this study, integrates the max-min residual energy routing and minimum total energy routing. It assigns a specific threshold for minimum residual energy, so the minimum energy required for transmission and receiver path chosen must be above this level. This study was continued by studying the routing for maximum network lifetime where the message series is not known a priori [9].

In 2004, Jae-Hwan Chang and Leandros Tassiulas proposed an algorithm to prolong WSNs lifetime through calculation of shortest cost path for routing [3]. This path uses connections cost between nodes, which represent both the communication power consumption rates and the remaining energy levels at the nodes end. However, this algorithm weakens the security of WSNs.

Energy-efficient Routing Algorithm to Prolong Lifetime (ERAPL) is proposed to solve the energy consumption problem [10]. Data gathering sequence is used in this algorithm to avoid overhead of reciprocal transmission and loop transmission between nodes.

Wang et al. provided a general framework for solving the Network Lifetime Maximization (NLM) problem of WSNs [11]. They used the Karush-Kuhn-Tucker (KKT) optimality conditions, which takes the influence of data rate, link access and routing into account to deduce analytical expressions of the general best network lifetime.

In 2012, an Energy Efficient Load-Balanced Clustering (EELBC) mechanism was proposed which concentrates on energy efficiency and load balancing by selecting optimal Cluster Head $(\mathrm{CH})$ [12]. This scheme shows improved power efficiency compared to previous studies in this area.

Teddy Mantoro et al. proposed two schemes to enhance energy efficiency in WSNs. The first scheme is to distribute multiple sensors as sinks in wireless sensor networks to balance the energy consumption among the wireless sensor nodes. The second is collecting sensed data using mobile sink nodes crossing WSN [13], [14].

Three routing parameters that affect the WSN lifetime proposed by [15], i.e. initial power of the nodes, the residual power in the nodes, and routing period. In addition, to improve the security of WSNs, they proposed an evaluation for applying trust and reputation models and their effects on the power consumption and reliability of the network 16].

A solution to maximize network lifetime was proposed by selecting optimal $\mathrm{CH}$ only and without considering the routing path [12], while also proposed in [15] as the main factors that influence energy consumption. Based on the current limitations above, this study proposed maximum network lifetime through optimal energy aware method for $\mathrm{CH}$ selection and electing energy aware routing path.

\section{RESEARCH MODEL}

One method to maximize WSNs lifetime is optimizing energy aware routing path from sensors that gather information till base station. This research optimizes energy aware mechanism to prolong network lifetime by minimizing power consumption.

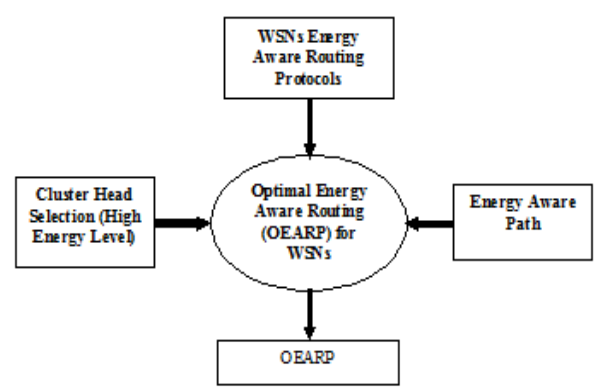

Figure 2. Model for Optimal Energy Aware Routing Path

As shown in Figure 2, this mechanism improves secure routing path by applying energy aware in selecting cluster 
head and routing path. $\mathrm{CH}$ is the sensor node, which has higher computing power than the other nodes available in the network. There may be more than one $\mathrm{CH}$ in a network. The $\mathrm{CH}$ performs actions like managing sensor nodes under its range and providing the information to base station. The base station in turn gives a routing command to $\mathrm{CH}$ based on the nodes energy level and its position. This information is passed to sensor node which needs to be in routing action.

Sensor nodes need to wait sink with cluster head in its communication range to send its data. In order to minimize this effect, many researchers are considering Multi-hop communication to reducing the sensor node waiting time. Many sensor nodes constrain resources causing the research community to face problems, such as low-power hardware design, routing algorithm, and optimization issues. In general, the main aim in extending the network lifetime in the real-time environment to charge or changing the sensor nodes batteries are almost an impossible and unfeasible activity.

\section{MATHEMATICAL MODEL}

In this section, the mathematical model for nodes and all network energy consumption during routing is presented. The energy consumed for node (i) is the total energy used when receiving data and energy used for transmission as follows:

$$
\mathrm{E}_{\mathrm{i}}=\mathrm{E}_{\text {transmit }}+\mathrm{E}_{\text {receive }}
$$

In details, the equation that represents energy (in Joules) consumed when the sensor receives a message of size $\mathrm{k}$ is

$$
E_{\text {receive }}=E_{\text {elec }} * k
$$

Where $\mathrm{E}_{\text {elec }}$ are electronics energy, and the energy consumed on sending a message of size $\mathrm{k}$ is

$$
E_{\text {transmit }}=E_{\text {elec }} * k+E_{\text {amp }} * r^{2} * k
$$

Where $\mathrm{r}$ is the sensor sensing range and $E_{a m p}$ is amplifier consumed energy [17]. So, the total energy consumed for the network is

$$
E_{\text {total }}=\sum_{i=1}^{n} E_{i}
$$

In this research the optimal energy calculation for sending packet from node $N_{i}$ to node $N_{j}$ can be estimated as follows:

$$
E_{\text {optimal }}=\operatorname{Cost}\left(N_{i}\right)+E\left(P_{\text {optimal }}\left(N_{i}, N_{j}\right)\right.
$$

Where $P_{\text {optimal }}$ is the optimal energy required for routing path between nodes $N_{i}$ and $N_{j}$.

\section{Optimal Energy Aware Routing Scheme}

There are still many challenges that data routing face such as high energy consumption during data transmission and security hand-shake of the routing path. OEARP considers energy consumption as an important issue. The following phase describes the OEARP scheme; network construction and routing.

\section{A. Phase I: Network Construction}

Once the sensors are deployed in a specific area, the network starts to construct itself. Cluster Heads (CHs) are elected for each cluster depending on the node's residual energy level, because the cluster head consumes more energy, its energy level must be more than the other nodes in the cluster. $\mathrm{CH}$ selection must be done periodically in each cluster once current $\mathrm{CH}$ energy level becomes less than any other node in its group. The following steps describe in detail network construction after deployment and how the cluster is created:

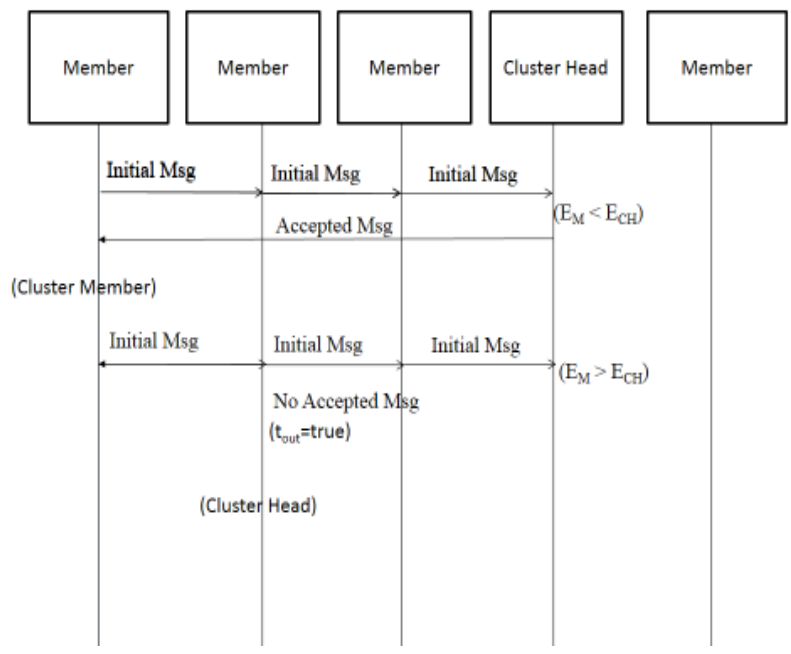

Figure 3. WSN clusters construction steps

Step 1: When a node comes up, it becomes in a state we can name it as initial-state, starts a timer and broadcasts an initial message, part of this message is energy level.

Step 2: When a cluster-head gets this initial message it responds with an accepted message immediately if its energy level is less or equal.

Step 3: When the initial-state node gets this message, it moves from initial-state to cluster member.

Step 4: If the initial-state node does not receive an accepted message during a certain period of time (times out), then it makes itself the cluster-head if it has bidirectional link to some neighbor, otherwise it remains in initial-state and repeats the steps again.

Step 5: Cluster heads are changed once their energy level becomes less than members.

Clustering plays a major role in wireless sensor networks which helps to extend the wireless sensor network lifetime. Mainly it involves grouping the sensor nodes into clusters which has cluster head for all the members in the network. The energy efficiency of cluster head persist variable initial energy rather than the normal node and it also reduces variance in initial energy level which helps to minimize the dead nodes in the sensor network.

Cluster head main job is to collect the data from the respective node, aggregate those data and send it to base station. Cluster head selection which mainly affects the wireless sensor network lifetime depends on the following three criteria: residual energy, number of neighbors and the distance from the base station to successive nodes.

Cluster transmits the data packets to base station without loss of data packet. When the energy level 
reduces abnormally during transmission of data packets to base station then that cluster head is changed to a cluster member. Since the cluster member doesn't receive any data from the cluster head for a while (idle state) after timing out it moves to initial state and repeats the steps again as shown in Figure 3. Each sensor node contains the uniform initial energy and it reports to the cluster head. In wireless sensor network, sensor energy levels are probabilistic rather than deterministic in each and every method. Figure 3 represents these steps.

Figure 4 shows the structure of WSN and Figure 5 displays the communication between network parts.

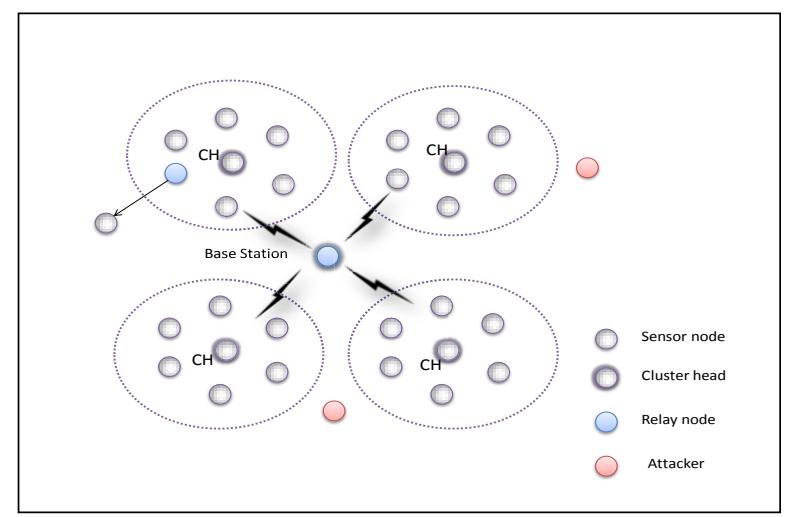

Figure 4. WSNs structure

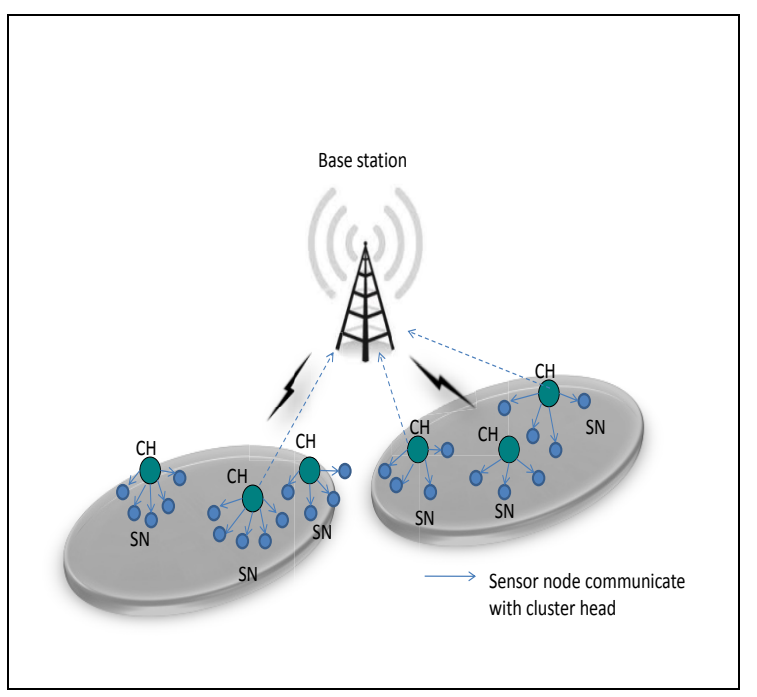

Figure 5. WSNs Communication

\section{B. Phase II: Routing Scheme}

Energy aware routing mechanism applies the following steps:

Step 1: Base station (BS) identifies the Cluster Heads (CHs) based on the energy. Once the cluster heads are selected, they send a formal handshake message to the neighboring nodes, similarly the neighboring node will send the acknowledge message to the cluster head.

Step 2: The Cluster Head maintains the information of the sensor node like id, position and energy level using a routing table. This information is shared to the Base Station.

Step 3: The ordinary sensor nodes (member) sends the collected information to the Cluster Head and maintains the acknowledgement from cluster head.
Step 4: Whenever a packet is transferred from one cluster to another cluster the $\mathrm{CHs}$ information is added in the packet for identification of other CHs.

Step 5: Communication will not happen without the knowledge of $\mathrm{CH}$, so every packet that needs to be transferred through is routed via a $\mathrm{CH}$.

Step 6: Before the packet is transferred to another cluster, the $\mathrm{CH}$ checks whether the destination node is under its group, if it is not in its group then it sends the packets to the Base Station. The base station has the information of $\mathrm{CHs}$ and nodes under it, and then it passes the packets to the relevant cluster head containing the destination node.

Step 7: In case a sensor node is on the move, it passes information like its direction and speed to the $\mathrm{CH}$. The base station knows the information from $\mathrm{CH}$, if source and destination sensor nodes are under range but with different $\mathrm{CHs}$, then it will communicate with the help of neighboring nodes.

Step 8: The packet transfer continues to occur even if the link with $\mathrm{CH}$ fails using neighboring nodes until it joins another to group.

All the previous steps of routing phase are summarized in Figure 6.

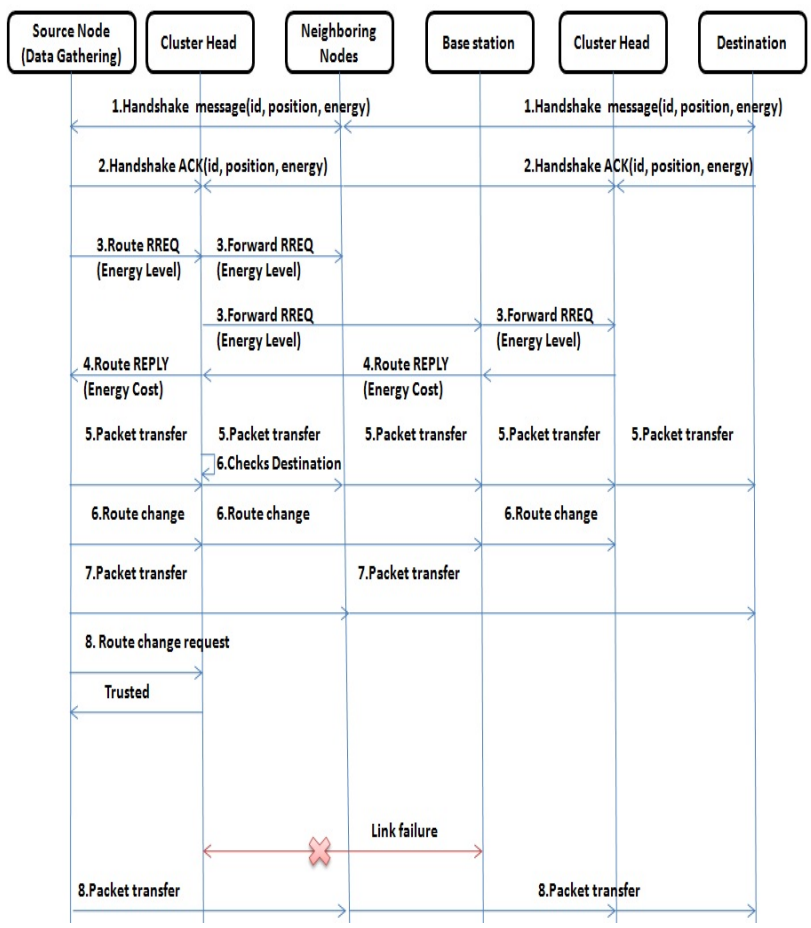

Figure 6. OEARP Routing phase steps

\section{EXPERIMENTS AND SIMULATION RESULTS}

This section describes OEARP simulation results in terms of energy consumption, and prolonged network lifetime compared with other protocols.

\section{A. Simulation Tool and Definitions of Parameter}

In this study, NS2 was used for WSNs simulation. Five experiments were carried out consisting different number of nodes randomly distributed over an area of 100 square units. Another assumption in this simulation, is that every node knows only its neighbors within the Radio Frequency $(\mathrm{RF})$ range. Each sensor node is assumed to 
have an initial energy of 100 Joules. A node is considered to be dead if its energy level reaches 0 Joules. Simulation parameters and default values used in the experiments are summarized in Table II.

TABLE II.

SIMULATION AND NETWORK PARAMETERS

\begin{tabular}{|c|c|c|}
\hline Parameter & Value & Comments \\
\hline $\mathrm{N}$ & $100,200,300,400,500$ & Number of nodes \\
\hline $\mathrm{E}_{0}$ & $100 \mathrm{j}$ & Node Initial Energy \\
\hline rx power & $1.0 \mathrm{rp}$ & Receive power \\
\hline tx power & $1.0 \mathrm{tp}$ & Transmit power \\
\hline $\begin{array}{c}\text { Data } \\
\text { packet }\end{array}$ & 50 bytes & Max packet in Ifq \\
\hline $\begin{array}{c}\text { max } \\
\text { Packet }\end{array}$ & $50000 \mathrm{bytes}$ & Max packet limit \\
\hline $\mathrm{I}_{\mathrm{t}}$ & $10.0 \mathrm{~s}$ & $\begin{array}{c}\text { Interval set for } \\
\text { packet transfer }\end{array}$ \\
\hline $\mathrm{B}$ & $512,1024,1500,2000,2500$ & Broadcast packets \\
& bytes & \\
\hline $\mathrm{P}_{\mathrm{opt}}$ & 0.05 & Optimal probability \\
\hline
\end{tabular}

In addition, this study assumed that all sensors are homogeneous, so each sensor has the same communication range. Also, sensors can only communicate with the neighbors within the communication range due to limited power. Multi-hop is required to communicate with farther ones.

\section{B. Experiments Results}

Since one of the essential constraints that affect the WSNs is battery limits and high energy consumption during transmission and receiving data (receiver), a dynamic WSN is simulated in our experiments. In these networks some sensors moves to idle state for a while if they do not receive any request from neighbors within a specific period of time. A sensor during idle state does not receive or transmit any data. After a certain timeout they wake up again.

In this research, experiments used WSNs that contain $100,200,300,400$, and 500 sensors respectively and deployed throughout 100 times 100 square network areas. The main objectives of these experiments were to measure the energy consumed during network life and the average execution time required for sending packet from source to base station after applying OEARP mechanism. Also a number of packets transmitted are calculated to measure network efficiency. Simulation results are summarized in Table III. Section VI.C and section VI.D describe in detail these results with graphical representation.

TABLE III.

SIMULATION RESULTS

\begin{tabular}{|c|c|c|c|c|}
\hline $\begin{array}{c}\text { Number } \\
\text { of } \\
\text { sensors }\end{array}$ & $\begin{array}{c}\text { Total energy } \\
\text { consumed ( } \\
\text { joules) }\end{array}$ & $\begin{array}{c}\text { Average } \\
\text { network } \\
\text { execution } \\
\text { time(ms) }\end{array}$ & $\begin{array}{c}\text { Number } \\
\text { of } \\
\text { packets }\end{array}$ & $\begin{array}{c}\text { Time till } \\
\text { the first } \\
\text { sensor } \\
\text { die }\end{array}$ \\
\hline 100 & 99 & 130.4 & 4608 & 114.65 \\
\hline 200 & 100 & 147.5 & 6144 & 128.89 \\
\hline 300 & 100 & 154.76 & 13500 & 140.35 \\
\hline 400 & 100 & 166.63 & 19000 & 153.34 \\
\hline 500 & 95 & 172.10 & 22500 & 159.07 \\
\hline
\end{tabular}

\section{Energy Consumption}

Long lifetime requirement of different applications and limited energy storage capability of sensor nodes has increased the need of reducing power consumption upon nodes. To increase sensors lifetime this mechanism is energy efficient.

In our simulation, initially, the energy level was set for each node equivalent to 100 Joules; however after we had run our simulation, the total energy used to transmit data did not exceed 100 joules. Energy consumption reaches its minimum value in experiments when 500 sensors were used as shown in Figure 7. In Figure 8, it shows that the network average execution time to transmit data packet for different network sizes. It is clear that average execution time for 100 sensors network did not have a large difference from average execution time for 500 sensors network which suggests significant improvement for small networks lifetime.

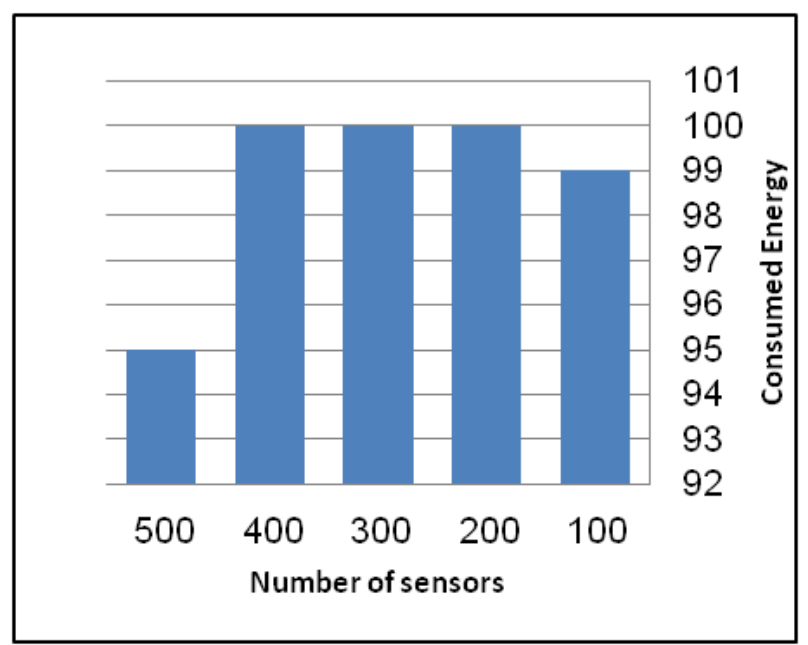

Figure 7. Energy consumed vs. number of sensors

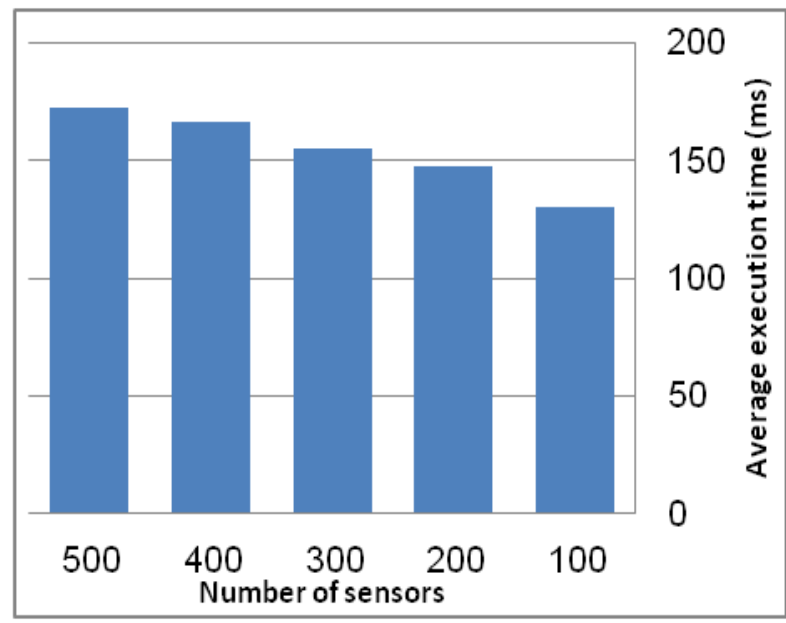

Figure 8. Average execution time vs. number of sensors

The communication range of a sensor node is relatively small when compared to the dimension of the whole wireless sensor network. Multi-hop is required to communicate with farther nodes, so, the average execution time remains the same when we have less number of sensors in network. Consider specific scenario with less sensors in a dimension 100 square network areas and it will take some time to communicate with other node. 
Sensors are increased then date transferring rate will be reduced because neighbor communicates frequently with less energy consumption. $\mathrm{CH}$ collects more information in a shorter period of time and sends it to base station. So, the average execution time of sensors in such a network is not too different from the average execution of 500 sensors.

Figure 9 shows the energy consumption during network execution for WSNs with 100, 200, 300, 400 and 500 respectively. The results show that energy conservation is achieved better using the proposed mechanism.

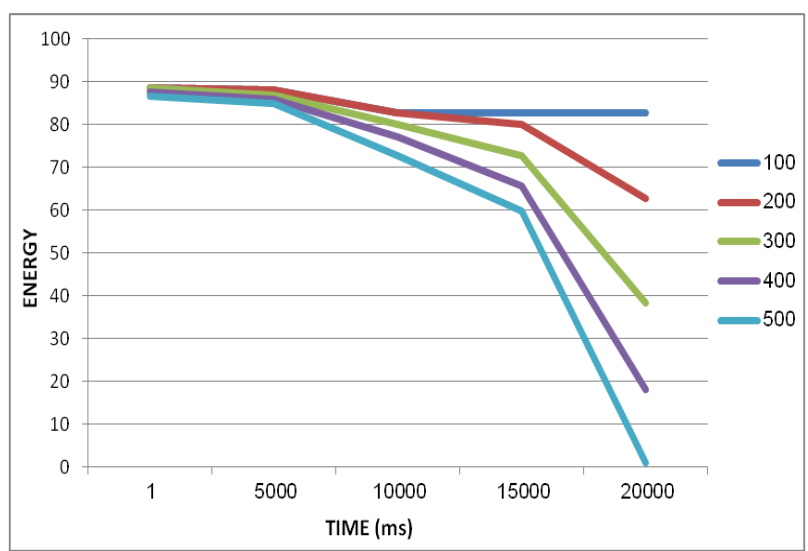

Figure 9. Energy consumption during network execution vs. number of sensors

\section{Network Efficiency}

Figure 10 presents the time passed before the first sensor died in different experiments. It is clear that the network efficiency improved through the use of OEARP especially for small networks which do not have large difference between it and large networks. This means applications using small networks with OERAP can be used widely once their efficiency is high and can remain alive for more time.

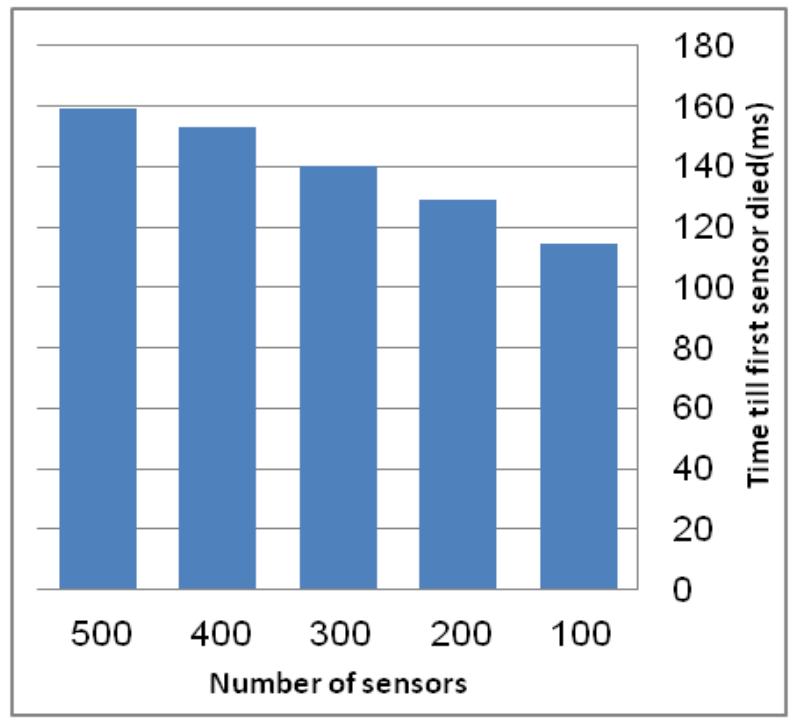

Figure 10. Time till first sensor died vs. number of sensors

The number of packets transmitted by the network during its life is a very important indicator that shows its efficiency. Figure 11 shows rapid increase in number of packets when the network size increased. Through comparison it is clear that a number of packets transmitted by network containing 500 sensors are $80 \%$ more than network with 100 sensors.

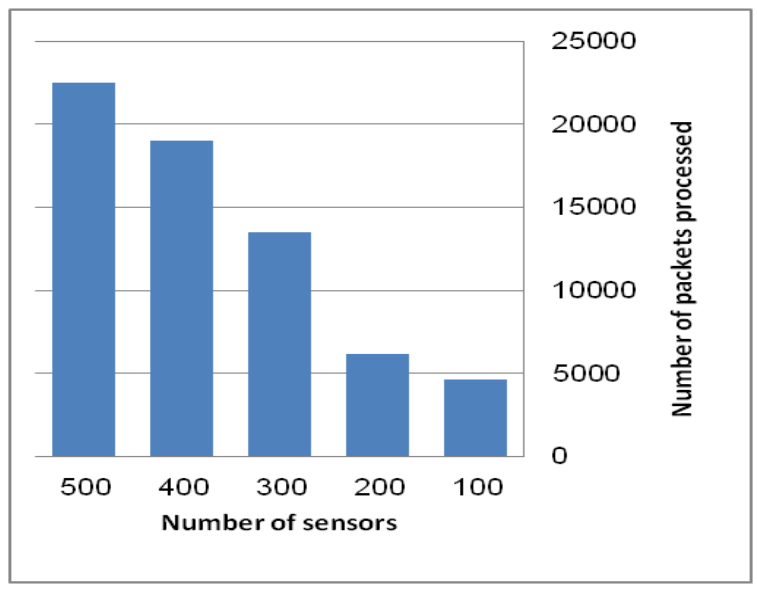

Figure 11. Number of packets transmitted vs. number of sensors

\section{E. Comparison with Known Mechanisms}

To prove efficiency of the proposed mechanism, it is compared with mechanisms described in [12]. The comparison factors are average execution time required to transmit data packet from source to destination, energy consumed and number of sensors that died during a specific period of time.

First, Table IV shows the results of average execution time for routing packets for different mechanisms with different network sizes. The results represented in Figure 12 shows OEARP in different network sizes have better results than other mechanisms, due to the optimal clustering and routing path; this means that a network can transmit a large amount of data before it dies when compared to other methods.

TABLE IV.

COMPARISON BETWEEN GLBCA, LBC, EELBC AND PROPOSED METHOD (OEARP) IN TERMS OF EXECUTION TIME

\begin{tabular}{|c|c|c|c|r|}
\hline No. of Sensors & EELBC & GLBCA & LBC & OEARP \\
\hline 100 & 150 & 170 & 160 & 130 \\
\hline 200 & 200 & 343 & 300 & 147 \\
\hline 300 & 310 & 400 & 375 & 154 \\
\hline 400 & 390 & 470 & 447 & 166 \\
\hline 500 & 450 & 500 & 489 & 172 \\
\hline
\end{tabular}

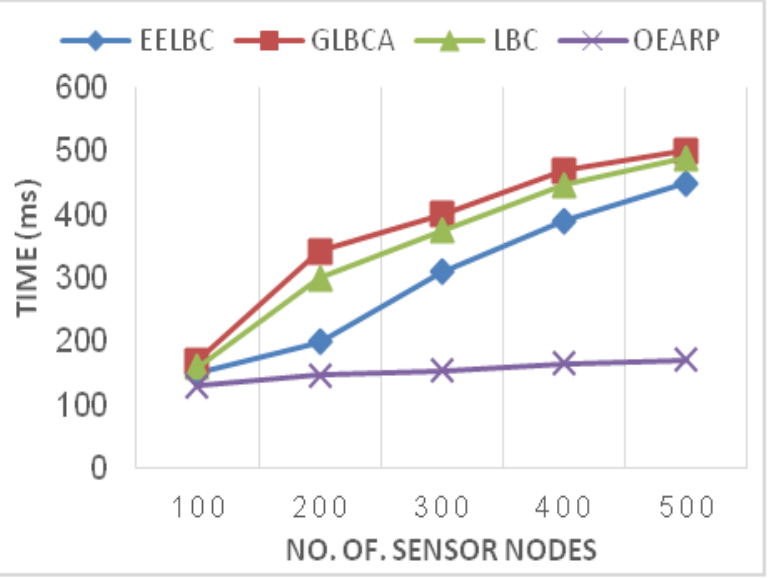

Figure 12. Comparison between GLBCA, LBC, EELBC and proposed method (OEARP) in terms of average execution time 
Energy consumption factor is the important factor that affects WSNs lifetime. Less energy consumption means that the network will stay alive for a longer time. Results shown in Table $\mathrm{V}$ and Figure 13 proved that OEARP consumes less energy than other methods for different network sizes.

TABLE V.

COMPARISON BETWEEN GLBCA, LBC, EELBC AND PROPOSED METHOD (OEARP) IN TERMS OF ENERGY CONSUMPTION

\begin{tabular}{|c|c|c|c|c|}
\hline Time & EELBC & GLBCA & LBC & OEARP \\
\hline 100 & 480 & 520 & 500 & 250 \\
\hline 200 & 980 & 1200 & 1000 & 400 \\
\hline 300 & 1480 & 1700 & 1500 & 550 \\
\hline 400 & 1980 & 2200 & 2000 & 800 \\
\hline 500 & 2880 & 3200 & 2980 & 1500 \\
\hline
\end{tabular}

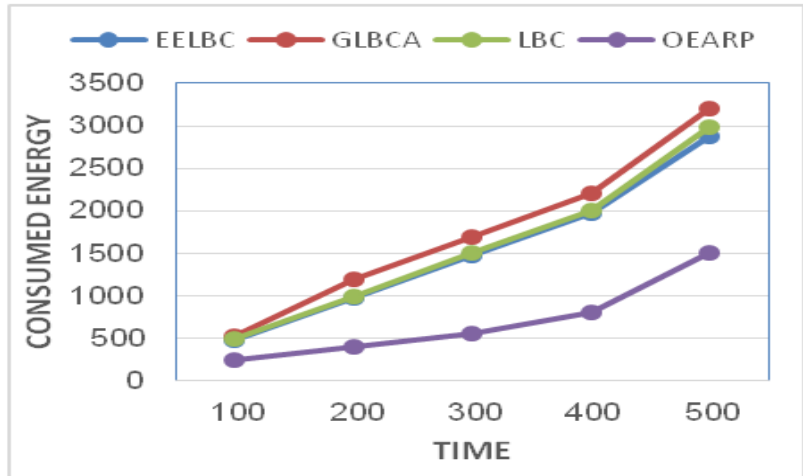

Figure 13. Comparison between GLBCA, LBC, EELBC and proposed method (OEARP) in terms of energy consumption

The last comparison factor is the number of sensors that died during specific periods of time. As shown in Table VI and Figure 14, OEARP has better performance than other mechanisms. During all simulation periods the number of sensors that died is less than 200 whereas it is more than 200 for all other mechanisms.

TABLE VI

COMPARISON BETWEEN GLBCA, LBC, EELBC AND PROPOSED METHOD (OEARP) IN TERMS OF NUMBER OF SENSORS DIED

\begin{tabular}{|c|c|c|c|c|}
\hline Time & EELBC & GLBCA & LBC & OEARP \\
\hline 100 & 200 & 200 & 200 & 110 \\
\hline 200 & 300 & 450 & 400 & 210 \\
\hline 300 & 400 & 475 & 450 & 290 \\
\hline 400 & 450 & 485 & 473 & 350 \\
\hline 500 & 470 & 490 & 478 & 360 \\
\hline
\end{tabular}

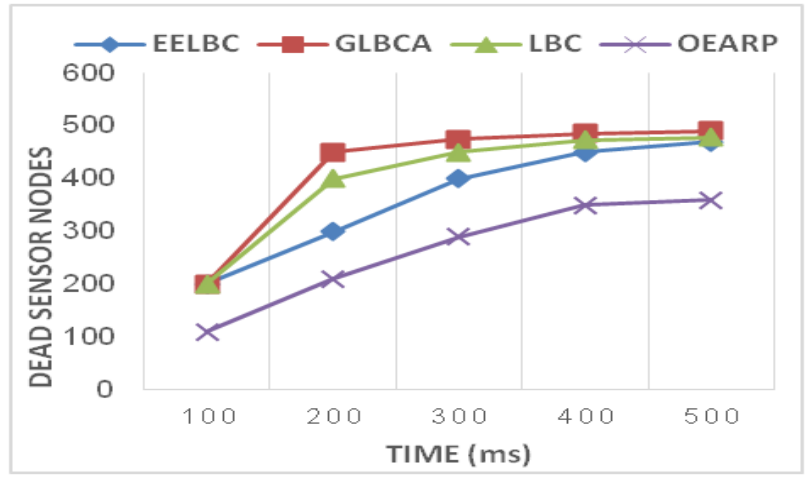

Figure 14. Comparison between GLBCA, LBC, EELBC and proposed method (OEARP) in terms of number of sensors died

\section{RESULTS AND DISCUSSION}

Simulation results demonstrate that OEARP achieved significant energy savings and improved network lifetime compared to GLBCA, LBC and EELBC. We present that OEARP obtains better network efficiency than other clustering methods based on routing protocols. As a result, this mechanism can be used with different WSNs sizes with high performance. From the results of the simulation and comparison with known methods, we can summarize the contribution of this paper in the following points:

- Experiment results have shown that total energy used to transmit data in the proposed model does not exceed 100 joules per sensor.

- Simulation results show that average execution time for small WSNs has little difference with average execution time for large WSNs, which means good improvement for small networks lifetime.

- The proposed model conserves energy. The result shows that energy consumption rate during execution time remains stable for different network sizes.

- OEARP improves the WSNs efficiency especially for small networks by increasing the time untill the first sensor dies and increasing the number of packets transmitted during network life.

- Comparing GLBCA, LBC, EELBC methods with OEARP shows in different network sizes OEARP has better results than other mechanisms, due to the optimal clustering and routing path; this means that the network can transmit large amount of data before it die rather than other methods.

- The execution time for OEARP is optimized so that it can perform the task in limited time period, the time taken to identify neighboring sensor nodes and perform packet transfer with less energy consumption.

- The energy consumption of the OEARP is much less compared to other mechanisms; this reduced energy consumption helps in maintaining the network for a longer time so that it can perform the task like transferring packets to the base station without affecting the computing power.

- OEARP mechanism reduces the number of dead sensor nodes in a network as it follows optimized energy consumption mechanism, with the help of this mechanism the load on individual sensor nodes is reduced, so many sensor nodes will remain active till the network lifetime.

\section{CONCLUSIONS}

The energy constraints and limited computing resources existing in sensor nodes causes major challenges in transmitting and processing data within the network. This study focused on how to minimize the node energy consumption, since it has been proven to be the essential part in wireless sensor networks. By considering the limitation of the wireless sensor networks, OEARP mechanism proposed to consume less energy when transferring data to the receiver which results in prolonged lifetime of the network. The main idea of this mechanism 
is the capability of selecting energy aware cluster head and routing path from nodes till the base station.

In OEARP before transferring packets the routes are optimized with minimum distance considering nodes energy level to the destination node are utilized for transferring packets, so that the power required to amplify the signal to the nodes far from the source node is reduced. This technique manages the battery backup for all nodes participating in a network. Simulation results prove the quality of this mechanism and its efficiency rather than other methods in the same area, energy consumption is minimized and number of sensors that died throughout the network life time is less compared to other mechanisms.

As a future work this mechanism can be improved by applying mobile sensors to solve the problem of isolated clusters due to dead sensors. Also the security issue can be measured by using trust and reputation techniques and authentication mechanism between network nodes along with reduced power consumption.

\section{REFERENCES}

[1] A. S. Alkalbani, A. M. Tap, and T. Mantoro, "Energy Consumption Evaluation in Trust and Reputation models for Wireless Sensor Networks", in Information and Communication Technology for the Muslim World (ICT4M), Morocco, 2013, pp.1-6.

[2] J. Shin, and C. Suh , "CREEC : Chain Routing with Even Energy Consumption", Journal Of Communications And Networks, Vol. 13, No. 1, 2011, pp.17-25. http://dx.doi.org/10.1109/JCN.2011. 6157247

[3] J. H. Chang, and L. Tassiulas, "Maximum Lifetime Routing in Wireless Sensor Networks", IEEE/ACM Transactions on Networking, Vol. 12, No. 4, 2004, pp.1126-1137. http://dx.doi.org/10.1109/TNET.2004.833122

[4] O. Zytoune, Y. Fakhri, and D. Aboutajdine, "A Fairly Balanced Clustering Algorithm for Routing in Wireless Sensor Networks", Sensor Review, Emerald, Vol. 30, No. 3, 2010, pp.242-249. http://dx.doi.org/10.1108/02602281011051434

[5] I. Almomani, and E. Almashakbeh, "A power-efficient secure routing protocol for wireless sensor networks", WSEAS Transactions on Computers Journal, 2010, Vol. 9, No. 9, pp.10421052 .

[6] J. N. Al-Karaki, and A. E. Kamal, "Routing techniques in wireless sensor networks: a survey", in Wireless Communications, IEEE, Vol. 11, No. 6, 2004, pp.6-28.

[7] O. Ahmed, A. Sajid, and M. A. Mehmood, "Comparison of Routing Protocols to Assess Network Lifetime of WSN", International Journal of Computer Science Issues (IJCSI), Vol. 8, No. 6, 2011, pp.220-224.

[8] C. K. Toh, "Maximum battery life routing to support ubiquitous mobile computing in wireless ad hoc networks", IEEE Communication Magazine, 2001, pp.138-147. http://dx.doi.org/ 10.1109/35.925682

[9] Q. Li, J. Aslam, and D. Rus, "Online power-aware routing in wireless ad hoc networks", In Proceedings of the 7th annual international conference on Mobile computing and networking, ACM, Italy, 2001, pp.97-107

[10] Y. Zhu, W. D. Wu, and J. Pan, "An energy-efficient data gathering algorithm to prolong lifetime of wireless sensor networks", Computer Communications, Vol. 33, No. 5, 2010, pp.639-647. http://dx.doi.org/10.1016/j.comcom.2009.11.008

[11] H. Wang, N. Agoulmine, M. Ma, and Y. Jin, "Network Lifetime Optimization in Wireless Sensor Networks", IEEE Journal On Selected Areas In Communications, Vol. 28, No. 7, 2010, pp.1127-1137. http://dx.doi.org/10.1109/JSAC.2010.100917

[12] P. Kuila, and P. K. Jana, "Energy Efficient Load-Balanced Clustering Algorithm for Wireless Sensor Networks ", Procedia
Technology, Vol 6, 2012, pp.771-777. http://dx.doi.org/10.1016/ j.protcy.2012.10.093

[13] T. Mantoro, H. S. Ali, and M. A. Ayu, "Energy Efficiency Mechanism Using Mobile-Based Fermat's Spiral in WSN", in 11th WSEAS International Conference on Applied Computer Science (ACS '11), Malaysia, 2011, pp.172-177.

[14] T. Mantoro, M. A. Ayu, H. S. Ali, W. Usino, and M. M. Kadhum, "Energy Efficiency Mechanisms Using Mobile Node in Wireless Sensor Networks", in Networked Digital Technologies (NDT), UAE, 2012, pp.536-550. http://dx.doi.org/10.1007/978-3-64230507-8_45

[15] A., S., Alkalbani, T., Mantoro, A., O., Md. Tap, "Improving the Lifetime of Wireless Sensor Networks Based on Routing Power Factors", in Networked Digital Technologies (NDT), UAE, 2012, pp.565-576.

[16] A. S. Alkalbani, A. M. Tap, and T. Mantoro, "Modified Reputation-Base Trust (MRT) for WSN", Journal of Theoretical and Applied Information Technology (JATIT), Vol. 56, No. 2, 2013, pp. 417-427.

[17] K. Sha and W. Shi, "Modeling the Lifetime of Wireless Sensor Networks", Sensor Letters, Vol.3, No.2, 2005, pp.126-135. http://dx.doi.org/10.1166/sl.2005.017

\section{AUTHORS}

Abdullah Said Alkalbani holds a Bsc and Msc Degree in Computer Engineering, from Arab Academy for Science \& Technology, Egypt in 2000 \& 2005 respectively. $\mathrm{He}$ is currently pursuing his $\mathrm{PhD}$ in Computer Science. $\mathrm{He}$ is working as Lecturer in University of Buraimi, Oman and as Research Assistant at the KICT, International Islamic University Malaysia. His research interests include Wireless Sensor Networks Security, Cryptography and Database Systems Security (email: abdullah.said@student.iium.edu.my).

Abu Osman Md. Tap awarded a PhD from University of Birmingham, UK and Msc from Iowa State University, Ames, Iowa, USA. Currently he is Professor in Mathematics at the Department of Computer Science, KICT, International Islamic University Malaysia. He has authored several research papers, several book and book chapters in the area of fuzzy logic and mathematics (email: abuosman@iium.edu.my).

Teddy Mantoro holds a $\mathrm{PhD}$, Msc and a Bsc, all in Computer Science. $\mathrm{He}$ was awarded a $\mathrm{PhD}$ from Department of Computer Science, the Australian National University (ANU), and Canberra, Australia in 2006. He is Vice Rector for Academic and Student Affairs (adinterim) and Dean of the Faculty of Science and Technology, Universitas Siswa Bangsa International, Jakarta, Indonesia. He has filed 4 (four) certificate Malaysian patents and published 3 (three) books and more than 100 papers, book chapters, journals and conference proceedings. His research interest is in pervasive/ubiquitous computing, context aware computing, mobile computing, location aware computing and intelligent environment. Prof. Mantoro is the founder and was the leader of the Integ Lab (Intelligent Environment Research Group) at KICT, International Islamic University Malaysia (IIUM), Kuala Lumpur, Malaysia. Integ Lab has received 43 medals since 2009 from national and international innovation technology competitions. He holds 4 (four) Malaysian patents under his name (e-mail: teddy@ieee.org).

Submitted 10 October 2014. Published as resubmitted by the authors 25 January 2015. 